\title{
The role of multimodal treatment in patients with advanced lung neuroendocrine tumors
}

\author{
Nicola Fazio ${ }^{1 *}$, Antonio Ungaro ${ }^{1}$, Francesca Spada ${ }^{1 *}$, Chiara Alessandra Cella ${ }^{1}$, Eleonora Pisa ${ }^{2 *}$, Massimo \\ Barberis $^{2 *}$, Chiara Grana ${ }^{3 *}$, Dario Zerini ${ }^{4 *}$, Emilio Bertani ${ }^{5 *}$, Dario Ribero ${ }^{5 *}$, Luigi Funicelli ${ }^{* *}$, Guido \\ Bonomo $^{7 *}$, Davide Ravizza ${ }^{8 *}$, Juliana Guarize ${ }^{9}$, Filippo De Marinis ${ }^{10}$, Francesco Petrella ${ }^{9}$, Ester Del \\ Signore $^{10}$, Giuseppe Pelosi ${ }^{11,12}$, Lorenzo Spaggiari ${ }^{9}$
}

${ }^{1}$ Unit of Gastrointestinal Medical Oncology and Neuroendocrine Tumors, ${ }^{2}$ Department of Pathology, ${ }^{3}$ Division of Nuclear Medicine, ${ }^{4}$ Division of Radiotherapy, ${ }^{5}$ Division of Hepatobiliopancreatic Surgery, ${ }^{6}$ Division of Radiology, ${ }^{7}$ Division of Interventional Radiology, ${ }^{8}$ Division of Endoscopy, ${ }^{9}$ Division of Thoracic Surgery, ${ }^{10}$ Division of Thoracic Medical Oncology, European Institute of Oncology, Milan, Italy; ${ }^{11}$ Department of Oncology and Hemato-Oncology, Università degli Studi di Milano, Milan; ${ }^{12}$ Inter-Hospital Pathology Division, Science and Technology Park, IRCCS MultiMedica, MIlan, Italy

Contributions: (I) Conception and design: N Fazio; (II) Administrative support: None; (III) Provision of study materials or patients: None; (IV) Collection and assembly of data: None; (V) Data analysis and interpretation: None; (VI) Manuscript writing: All authors; (VII) Final approval of manuscript: All authors.

*ENETS Center of Excellence for GEP NEN.

Correspondence to: Nicola Fazio, MD, PhD. Unit of Gastrointestinal Medical Oncology and Neuroendocrine Tumors, European Institute of Oncology, Via Ripamonti 435, 20141 Milan, Italy. Email: nicola.fazio@ieo.it.

\begin{abstract}
Lung neuroendocrine tumors (NETs) comprise typical (TC) and atypical carcinoids (AC). They represent the well differentiated (WD) or low/intermediate grade forms of lung neuroendocrine neoplasms (NENs). Unlike the lung poorly differentiated NENs, that are usually treated with chemotherapy, lung NETs can be managed with several different therapies, making a multidisciplinary interaction a key point. We critically discussed the multimodal clinical management of patients with advanced lung NETs. Provided that no therapeutic algorithm has been validate so far, each clinical case should be discussed within a NEN-dedicated multidisciplinary team. Among the systemic therapies available for metastatic lung NETs everolimus is the only approved drug, on the basis of the results of the phase III RADIANT-4 trial. Another phase III trial, the SPINET, is ongoing comparing lanreotide with placebo. Peptide receptor radionuclide therapy and chemotherapy were not studied within phase III trials for lung NETs, and they have been reported to be active within retrospective or phase II prospective studies. Temozolomide and oxaliplatin are two interesting chemotherapeutic agents in lung NETs. While some European Institutions were certificated as Centers of Excellence for gastroenteropancreatic NENs by the European Neuroendocrine Tumor Society (ENETS), an equivalent ENETS certification for lung NENs does not exist yet. Ideally a lung NEN-dedicated multidisciplinary tumor board should include NEN-dedicated medical oncologists, thoracic medical oncologist, thoracic surgeons, pathologists, interventional radiologists, endocrinologists, radiotherapists, interventional pneumologists, nuclear physician.
\end{abstract}

Keywords: Lung NET; lung carcinoid; typical carcinoid; atypical carcinoid (AC); bronchopulmonary carcinoid

Submitted Feb 27, 2017. Accepted for publication May 19, 2017.

doi: $10.21037 /$ jtd.2017.06.14

View this article at: http://dx.doi.org/10.21037/jtd.2017.06.14 


\section{Introduction}

Lung neuroendocrine malignancies represent around $25 \%$ of all lung cancers. Unlike gastroenteropancreatic (GEP) neuroendocrine neoplasms (NENs), that are mainly well differentiated (WD), lung NENs are for the vast majority poorly differentiated (PD). Small cell lung cancer (SCLC) represents roughly $20 \%$ of all lung cancers, large cell neuroendocrine carcinoma (LCNEC) $3 \%$, atypical carcinoid (AC) $0.3 \%$ and typical carcinoid (TC) $2 \%(1-3)$. SCLC and LCNEC represent the PD or high grade category, whereas $\mathrm{AC}$ and TC are the WD or low/intermediate grade category $(4,5)$. In other words, extrapolating from the GEP tract, the former can be defined as neuroendocrine carcinomas (NECs) and the latter as neuroendocrine tumors (NETs) (6). From a therapeutical point of view in advanced disease the therapeutic approach is different between high and low/intermediate grade lung NETs. In the high grade category surgery has a limited role, whereas chemotherapy and to a lesser extent external beam radiotherapy are routinely considered; biological therapies have not been reported to be effective. By contrast in the low/intermediate grade category several systemic therapies can be considered, including chemotherapy, biotherapy, radionuclide therapy, but also locoregional procedures, such as palliative surgery, radiotherapy and interventional radiology procedures, mainly on the liver.

To have a right idea about the therapeutical approach to lung NETs two main features should be considered. First, while in clinical practice of a thoracic medical oncologist lung NETs represent a very rare entity, they are quite frequent in the clinical practice of a NEN-dedicated medical oncologist, representing around one third of all low/intermediate grade NENs (Figure 1). Second, while in SCLC and LCNEC a thoracic medical oncology has a dominant role compared with multidisciplinarity, in lung NETs multidisciplinarity is relevant. Lung NETs should be managed in a similar manner than low/intermediate grade GEP NENs, which are also defined as NETs in accordance with WHO 2010 GEP NEN classification (6). While this is difficult to be found in real world clinical practice, it can be obtained in some NEN referral centers, where the case of a patient with a lung NET is usually discussed within a multidisciplinary team, potentially including medical oncologists, surgeons, pathologists, radiologists, endocrinologists, radiotherapists, pneumologists, endoscopists.

For several years the European Neuroendocrine Tumors
Society (ENETS) has been certificating Centers of Excellence (CoE) for GEP NENs, on the basis of a number of requirements starting from the threshold of $>80$ new GEP NEN-patients per year. Unfortunately an ENETS CoE certification for lung NENs does not exist yet. Therefore lung NET patients clinical management can be various even in NEN referral centers.

In this paper we will critically discuss the multidisciplinary therapeutical approach to patients with lung NETs, focusing on the advanced stage and criteria to choose therapy.

\section{General criteria for choosing therapy in patients with advanced lung NETs}

Evidence about therapy in advanced lung NETs is quite poor, therefore we are far from having a therapy that can be defined as a gold standard, apart from radical surgical resection in locally advanced lung NET. In the vast majority of cases, particularly in metastatic disease, several different therapies could be considered in the same clinical setting. More in general two main categories can be evaluated, comprising locoregional and systemic therapies. Among the former there are palliative surgical resection of the primary site or metastatic disease, palliative external beam radiotherapy, palliative interventional radiology procedures including liver transarterial embolization (TAE), thermoablation radiofrequency (TARF) and liver transarterial radioembolization (TARE) with ${ }^{90}$ Yttrium. The latter category comprises somatostatin receptor 2 (SSTR-2)directed therapies, including somatostatin analogs (SSAs) and Peptide Receptor Radionuclide Therapy (PRRT), molecular targeted agents (MTAs) like Everolimus, chemotherapy (several regimens) and interferon (IFN).

It is clear that this pool of therapies potentially applicable to a patient with lung NETs is tremendously heterogeneous, in terms of feasibility/reproducibility, level of evidence, regulatory rules and accessibility to clinical trials. Likewise it is easily deducible that a single specialist, although skilled, cannot adequately manage the patient over the whole course of his/her disease.

Therefore it would be ideal that every single case was discussed within a multi-specialist team and that this team comprised lung NEN-skilled specialists. For each speciality a NEN-dedicated figure should be designate to be part of the team. The routine interaction with other figures dedicated to lung NEN increases the level of awareness about these diseases and expertise.

An algorithm of criteria for choosing therapy 
A

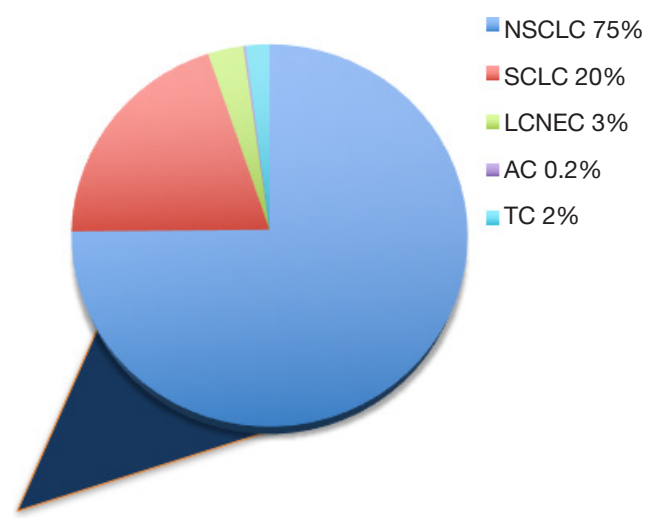

Thoracic medical oncologist's view
B

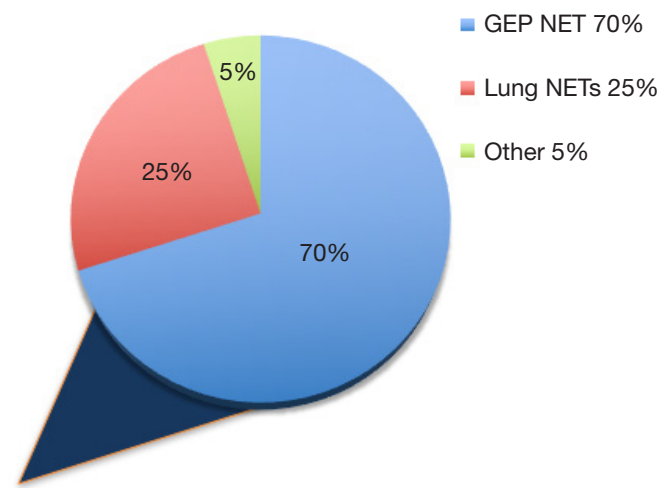

NEN-dedicated medical oncologist's view

Figure 1 Lung NETs in the medical oncologist's view. (A) Frequence of lung NETs (also named as carcinoids) in thoracic medical oncologist's clinical practice; (B) frequence of lung NETs in the NEN-dedicated medical oncologist's clinical practice. NEN, neuroendocrine neoplasm; NETs, neuroendocrine tumors; NSCLC, non-small cell lung cancer; SCLC, small cell lung cancer; LCNEC, large cell neuroendocrine carcinoma; AC, atypical carcinoid; TC, typical carcinoid; GEP, gastroenteropancreatic.

\section{Evidence}

\section{Regulatory authorities}

Recommendations/guidelines

\section{Logistics/Expertise/Experience}

Clinical trials

Figure 2 Criteria for choosing therapy in patients with advanced lung NETs. NETs, neuroendocrine tumors.

should start from evidence, critically analyzing the literature and correlating it to the clinical scenario to be managed (Figure 2). Sometimes approvals by regulatory authorities can condition strongly the therapeutic choice. Recommendations by experts in the field and/or guidelines are usually checked. Single center's or specialist's expertise, especially for technical procedures, as for instance interventional radiology procedures or surgery, is another point potentially conditioning the therapeutic choice. Again logistics sometimes can have a role, for instance for PRRT, that is related to the expertise of a limited number of Centers. Last but not least clinical trials availability can condition the choice of a therapy.

\section{Multidisciplinary management of patients with advanced lung NETs}

The right treatment is strictly correlated to the right diagnosis. For this aim the pathologist's role is crucial. It should be considered that sometimes even expert pathologists can run into objective difficulties related to the poorness or inadequacy of the material to analyse. From a therapeutic standpoint it is essential to rule out first a non-small cell lung cancer (NSCLC) and thereafter the PD forms of NENs, such as SCLC and LCNEC (Figure 3). Therapeutic options are clearly different between non neuroendocrine and neuroendocrine lung cancers, and between PD and WD lung NENs. For instance SSTR2-directed therapies can have an impact in WD but not in PD lung NENs. Although the two extremes, TC and SCLC, can be objectively distinguished each other, at least in surgical specimens (5), more difficult it can be separating AC from LCNEC, and in this case subjectivity of the pathologist can play a role to apply the conventional criteria. It should kept in mind that this may have practical clinical implications, both in terms of over-treatment (e.g., slow-growing LCNEC uselessly treated with intensive chemotherapy) and undertreatment (fast-growing AC inadequately treated with biotherapy).

Difficulties in recognizing mitosis and necrosis in the group of lung NETs can make difficult separating TC from AC. Once again it may have practical clinical implications, 


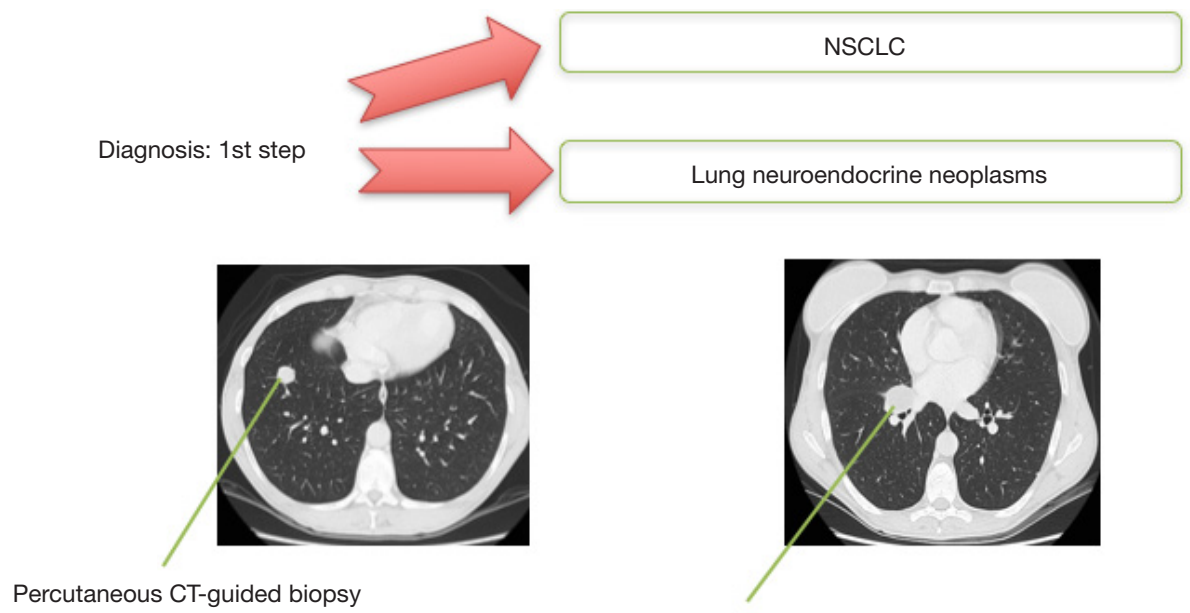

EBUS-TBNA: endobronchial ultrasound (EBUS) transbronchial needle aspiration (TBNA)

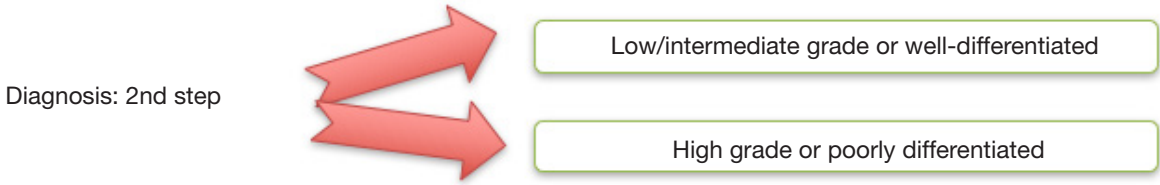

Figure 3 Lung nodule: step by step diagnostic work-up. NSCLC, non-small cell lung cancer.

given that some expert consensus, like that from the ENETS, made recommendations specifically related to TC and AC (7). However in clinical practice it is essential to be sure that the tumor is WD and $\mathrm{Ki}-67$ is relatively low rather than exactly knowing that it is TC or AC. Indeed therapeutic options are usually similar for TC and AC including both biotherapies and non platinum/etoposide chemotherapy (8).

To reduce these potential problems that a pathologist can encounter in diagnosing a lung NET, source and type of biological material may have a role. In metastatic stage preferably a sample from a lung lesion should be obtained, better if surgical. Unfortunately for lung NETs with synchronous distant metastases the surgical sample of the primary tumor could be not available. Furthermore sometimes diagnosis is based on cytology rather than histology from the primary tumor site or from a regional node, by means of a transbronchial needle aspiration (TBNA) endobronchial ultrasound (EBUS). Less commonly just the histological sample from the distant metastasis site is available, more commonly from the liver. In this case to be able to conclude for a lung NET it is important to correlate pathology information, including immunohistochemistry (IHC), with clinical picture and clinical history.
Although diagnosis is crucial it is not enough to decide about a therapy. Knowing about tumor and patient characteristics is needed. More in general it is clinically relevant to separate fast-growing/highly aggressive lung NENs, the behavior and therapy of which would merge with that of classical SCLC, from relatively slow-growing lung NENs for which therapeutic approach is completely different, mainly based on integration of several different therapies and use of biotherapy. To this aim tumor should be defined in terms of grading, morphological and functional imaging and clinical behavior. The current WHO classification (9), is not completely consistent with the subsequent clinical management of lung NET patients, particularly for metastatic disease, where AC and LCNEC can be difficult to recognize, as their definition is based on surgical specimens, often missing in this setting (10). Notably, a suspicion of LCNEC could be raised only for biopsy specimens (cytology is deemed unsuitable) (10) (Figure 4).

Once obtained the diagnosis of a lung NET an SSTR2-related imaging is needed, since most lung NETs express SSTR-2. A positron emission tomography (PET)-computed tomography (CT) with ${ }^{68}$ Gallium-DOTA-peptide is highly recommended, as it is much more sensitive than somatostatin receptor scintigraphy (SRS) (11). Furthermore 


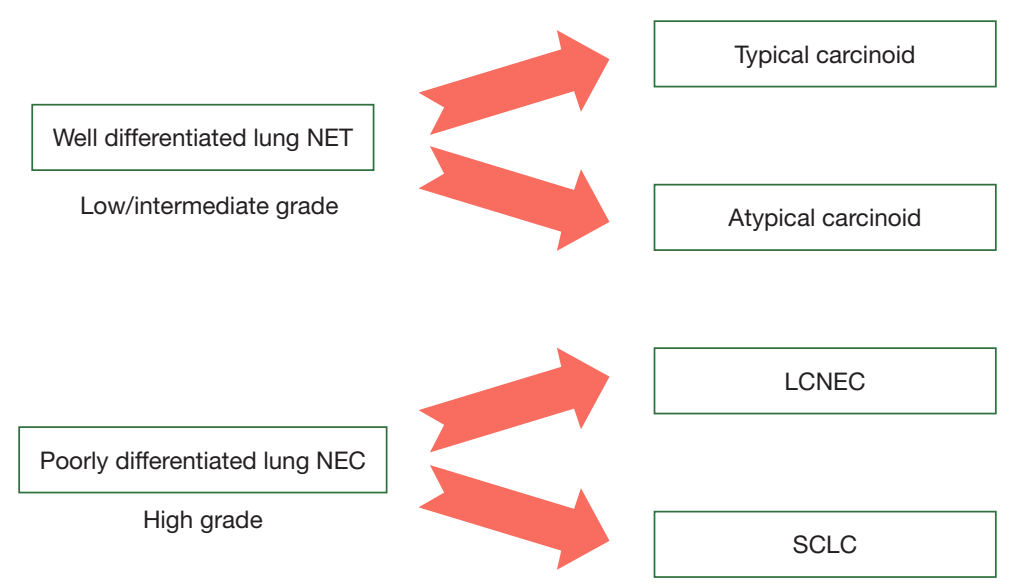

Figure 4 Terminology of lung neuroendocrine malignancies. NET, neuroendocrine tumor; NEC, neuroendocrine carcinoma; LCNEC, large cell neuroendocrine carcinoma; SCLC, small cell lung cancer.

Table 1 Characterization of disease in lung NETs

\begin{tabular}{llll}
\hline Type of lung NET & Morphological imaging & Functional imaging & Circulating markers \\
\hline Well differentiated & Total-body CT scan & ${ }^{68}$ Ga-PET/CT-DOTA-peptide & CgA \\
Or very low Ki-67 & & \\
Or low grade & & \\
Or TC & ${ }^{68}$ Ga-PET/CT-DOTA-peptide + ${ }^{18}$ FDG-PET-CT & CgA + NSE \\
Well/moderately differentiated & Total-body CT scan & \\
Or intermediate Ki-67 (e.g., 3-20) & & \\
Or AC & & \\
Poorly differentiated & & \\
Or high Ki-67 (e.g., $>20 \%)$ & & \\
Or LCNEC/SCLC & & \\
\hline
\end{tabular}

NETs, neuroendocrine tumors; TC, typical carcinoid; AC, atypical carcinoid; LCNEC, large cell neuroendocrine carcinoma; SCLC, small cell lung cancer; CT, computed tomography; Ga, gallium; PET, positron emission tomography; FDG, fluorodeoxyglucose; CgA, chromogranin-A; NSE, neuron specific enolase.

the Gallium-PET can detect bone metastases better than CT-scan and bone scintigraphy (12). For detecting ${ }^{68} \mathrm{Ga}-$ $\mathrm{PET} / \mathrm{CT}$ has been reported more sensitive than ${ }^{18} \mathrm{~F}-\mathrm{FDG}$ PET/CT, whereas in AC ${ }^{18} \mathrm{~F}-\mathrm{FDG}$ PET/CT can have a role in the preoperative work-up $(13,14)$. In the Table 1 is reported a scheme for characterization of lung NENs (Table 1) that we usually apply within our multidisciplinary team. Moving from indolent to fast-growing NENs SSTR-2 imaging is gradually replaced by the metabolic $\left({ }^{18} \mathrm{FDG}\right)$ one and plasma Chromogranin-A (CgA) by neuron specific enolase (NSE).
In a recently published article about a retrospective series of 105 patients with lung NETs managed in a British tertiary referral centre in the period 1998-2012 pathological classification in TC or AC was possible in 82 (15). Surprisingly ${ }^{68} \mathrm{Ga}$-PET-CT-Dotatate showed a similar sensitivity than SRS, and overall survival for TC was not significant different from that of AC. Furthermore a $10 \%$ $\mathrm{Ki}-67$ threshold was correlated with a statistically significant difference in survival.

The Ki-67 labeling index (LI) is not used to classify lung NENs in accordance with the WHO lung NEN 
classification (9). However it has been reported having a significant prognostic value when used in combination with mitotic index and necrosis (6). The combination of these three variables produced an effective grading system, identifying three groups (Ki-67\% cutoffs of these groups were: G1 <4, G2 4-25, G3 225). For clinicians Ki-67 remains a helpful parameter to decide about therapy; of course it should be correlated with all the other parameters as above mentioned in the manuscript.

Clinical picture can guide the therapeutic choice when there is a symptomatic progression or a paraneoplastic syndrome, that it is usually combined with a TC and more commonly it is a carcinoid syndrome (7). In these cases syndrome control represent a priority and can be obtained with SSAs +/- systemic anti-tumor therapies +/- debulking procedures. It should be discussed within a multidisciplinary team to involve all the expertises, on the basis of severity of syndrome and tumor burden

\section{Systemic therapies in lung NETs}

\section{Somatostatin analogs}

While the anti-syndromic role of SSAs utilized in clinical practice, such as octreotide and lanreotide, is well recognized, their antiproliferative role in lung NETs is debated, since the level of evidence about it is very low. However, clinicians usually extrapolate evidence existing in GEP NETs and they often use octreotide or lanreotide as first-line therapy in patients with advanced lung NETs, provided that it is slow-growing, with a limited tumor burden and in asymptomatic patients. A randomized, double-blind, phase 3 study (SPINET, NCT02683941) evaluating the efficacy and safety of lanreotide-autogel $v s$. placebo is ongoing in patients with well-differentiated, metastatic and/or unresectable, TC and AC.

\section{Everolimus}

No specific phase III trial for lung NETs was conducted so far, whereas the results of a randomised three-arm phase II trial enrolling just thoracic carcinoids have been presented at the last ESMO meeting (16). However, a large population of metastatic lung NETs was included in the RADIANT-4 study, comparing everolimus $10 \mathrm{mg} /$ day plus best supportive care versus placebo plus best supportive care in patients with advanced progressing non-functioning NETs of gastrointestinal or lung origin (17). In this study
90 out of 302 patients enrolled (30\%) had a lung NET (everolimus, $\mathrm{n}=63$ and placebo, $\mathrm{n}=27$, considering the $2: 1$ randomization). This is the largest lung NETs series ever included in a phase III trial. Median PFS by central review in the lung subgroup was 9.2 (95\% CI: 6.8-10.9) months in everolimus arm vs. $3.6(1.9-5.1)$ months in placebo arm (HR, 0.50; 95\% CI: 0.28-0.88). Data of efficacy and safety were consistent with those of the overall RADIANT-4 population (18).

The three-arm, randomized phase 2 LUNA trial compared pasireotide, an investigational SSA, in combination with everolimus vs. everolimus alone and pasireotide alone in patients with TC, AC or thymic carcinoids. A total of 112 patients were included. The LUNA study achieved the preplanned statistical objective of a 9-month PFS rate $>20 \%$ in all three arms, supporting the efficacy of everolimus in lung NETs.

Everolimus had been reported active and potentially effective also in a post-hoc analysis of the RADIANT-2 trial regarding a subgroup of lung NETs combined with carcinoid syndrome (18). Unlike the lung NET subgroup from the RADIANT-4, the RADIANT-2 lung NET subgroup had a lower number of patients and an imbalance between arms ( $33 v s .11$, for everolimus + octreotide LAR vs. placebo + octreotide LAR, respectively), due to the lack of stratification.

On the basis of the RADIANT-4 results everolimus was recently approved for treatment of patients with advanced lung NETs; this is the first agent ever approved for advanced lung NETs. Although this represents a good step forward in treatment of lung NETs, no absolute indication about timing and clinical setting can be drawn from the literature; once again the multidisciplinary interaction discussing the aformentioned clinical algorithm should be applied.

Other than RADIANT-4 and -2 everolimus was investigated within other minor studies $(19,20)$ and it is one of the agent most widely studied in patients with lung NETs (Table 2).

\section{Peptide receptor radionuclide therapy (PRRT)}

Evaluating the SSTR-2 functional expression by means of a Gallium-PET is helpful for at least three reasons, such as staging, prognostic information and prediction of response to PRRT. The feasibility of PRRT should be evaluated by a NEN-dedicate nuclear physician and indication to PRRT shared within the multidisciplinary team. So far no 
Table 2 Studies including patients with thoracic NETs receiving everolimus

\begin{tabular}{lccccc}
\hline Name of the study & Experimental arm & Control arm & Type of study & $\begin{array}{c}\text { Slung subgroup } \\
\text { characteristics }\end{array}$ & $\begin{array}{c}\text { Pts with lung NET } \\
\text { receiving everolimus }\end{array}$ \\
\hline RAMSETE & EVE & No & Phase II & Non-functioning & $22 / 73$ \\
RADIANT-2 & EVE + OCT LAR & OCT LAR + PLACEBO & Phase III & Carcinoid Syndrome \\
RAD-ITMO & EVE + OCT LAR & No & Phase II & First line \\
RADIANT-4 & EVE & PLACEBO & Phase III & Non-functioning \\
LUNA & PAS vs. EVE vs. & No & Rand. phase II & Mixed & $90 / 302$ \\
& PAS/EVE & & & $83 / 121$ \\
\hline
\end{tabular}

EVE, everolimus; OCT, octreotide; LAR, long acting repeatable; PAS, pasireotide; Rand., randomized.

Table 3 Studies of PRRT including more than 10 patients with advanced lung NETs

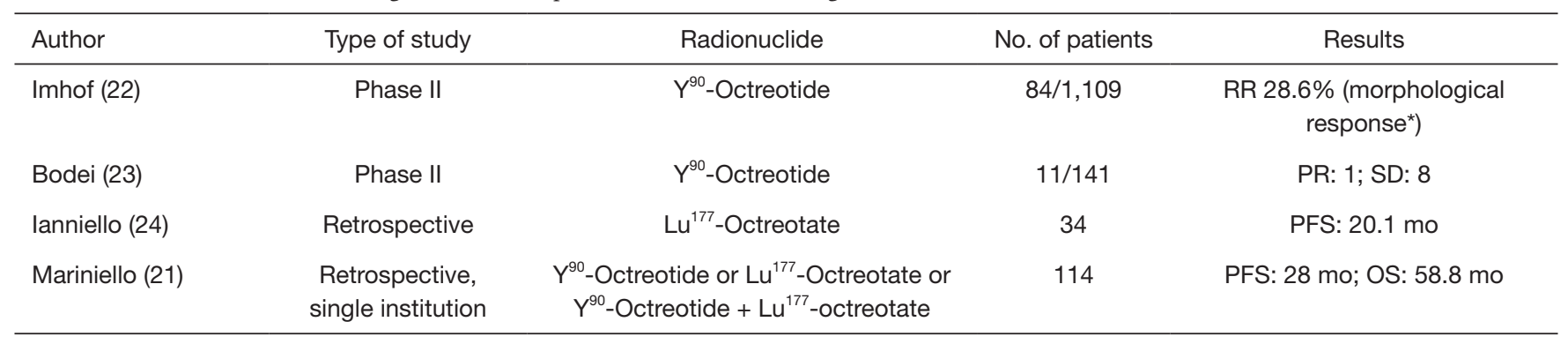

PFS, progression free survival; OS, overall survival; RR, response rate; mo, months.

prospective studies were performed with PRRT specifically in lung NETs. A very low number of patients with lung NETs were included in retrospective or phase II studies. The only large series of lung NETs treated with PRRT was recently published by Mariniello et al. (21). This is a retrospective single-center series of 114 patients with pretreated advanced lung NETs, from the European Institute of Oncology, in Milan, who received three different PRRT protocols: ${ }^{90} \mathrm{Y}$-octreotide versus ${ }^{177} \mathrm{Lu}$-octreotate versus ${ }^{90}$ Y-octreotide $+{ }^{177} \mathrm{Lu}$-octreotate. Median PFS and median OS were 28 and 59 months, respectively, with a response rate up to $39 \%$ with the ${ }^{90} \mathrm{Y} /{ }^{177} \mathrm{Lu}$ combination treatment (Table 3).

\section{Chemotherapy}

With regard to chemotherapy several drugs have been reported to be active even though no absolute data about efficacy in lung NETs exist so far (7). A combination of cisplatin or carboplatin with etoposide, that is the most used regimen in SCLC, has been used also in lung NETs (25), with around $20 \%$ of response rate (RR) and 7 months of progression free survival (PFS). This type of data should be interpreted with caution since they come from retrospective analyses, with a low number of patients and without a lung NEN-dedicated central pathology review. In an old study by Fjiallskog et al. published in 2001, 27 patients with a WD NET showed $33 \%$ of RR, which was similar to the $40 \%$ of RR of the 9 patients with a PD NEC; all patients with WD had a fast-growing disease at baseline. This means that some lung NETs can have a clinical behavior similar to those of the lung NECs and therefore they can respond to platinum/ etoposide chemotherapy in a similar manner. In these cases an inadequate diagnosis cannot be excluded without a strict pathology review of a sample with a reliable biological material by a skilled lung NEN-dedicated pathologist; and even in cases where the diagnosis of TC or AC were confirmed after this type of pathology review other factors should be known, including Ki-67 LI, functional imaging and rate of baseline radiological and clinical evolution. Unfortunately this type of information is often missing in the few published studies reporting chemotherapy in lung NETs.

Currently, two chemotherapeutic agents are promising 
Table 4 Temozolomide-based and oxaliplatin-based chemotherapy in advanced lung NETs

\begin{tabular}{|c|c|c|c|c|}
\hline Author & Regimen & Prospective/retrospective & No. of patients & Results (mo) \\
\hline Bajetta (27) & Capecitabine and Oxaliplatin & Phase II & $5 / 40$ & PFS: 20 \\
\hline Spada (28) & Oxaliplatin based & Retrospective & $19 / 78$ & PFS: 8 \\
\hline Crona (29) & Temozolomide & Retrospective & 31 & PFS: 5.3 \\
\hline Kunz (31) & Oxaliplatin based + Bevacizumab & Phase II & $42 / 76$ & PFS: 19 \\
\hline
\end{tabular}

mo, months.

in lung NETs, such as temozolomide and oxaliplatin (Table 4). Temozolomide is a derivative oral agent of the progenitor streptozotocin, belonging to the alkylating category. It has been investigated mainly in pancreatic NETs and data about lung NETs come from studies, often retrospective, including mixed populations of NETs and NECs $(25,29,32,33)$.

Oxaliplatin has been reported active and potentially effective in retrospective analyses of patients with metastatic lung NETs alone or lung NETs mixed with other primary sites, treated with GEMOX, CAPOX or FOLFOX regimens $(26,28)$.

Temozolomide and oxaliplatin chemotherapy represent two good options for medical oncologists. The former can be proposed as single agent or in combination, usually with capecitabine, with all the advantage of oral regimens for patients. The latter is well known to the vast majority of medical oncologists, who are confident with several combination regimens, in particular fluoropyrimidinesbased.

\section{Concluding remarks}

Lung NETs represent a very rare entity for thoracic medical oncologists, whereas they are quite common for NEN-dedicated medical oncologists. Clinical management of lung NET patients is similar to that of GEP NET patients, mainly based on a multidisciplinary diagnostictherapeutic management. However, while dedicated tumorboard for GEP NETs exist in several International Referral Centers and some of them were certificated as ENETS CoE for GEP NET on the basis of specific requirements, similar tumor boards are very rare for lung NETs and there is not an equivalent ENETS certification yet. European Institute of Oncology (IEO) is one of the ENETS CoE for GEP NENs; at the IEO lung NET patients clinical cases are usually discussed within the weekly meeting for the NEN-dedicated multidisciplinary team. Provided that no specific sequence or combination has been validated so far, then a therapeutic algorithm is discussed and shared among the various specialists. Thoracic surgeons, interventional pneumologists, thoracic medical oncologists and radiotherapists, are not fixed members of the NENdedicated tumor board, but they are involved as needed to discuss lung NET clinical cases. A diagnostic-therapeutic multimodal clinical management is relevant for patients with advanced lung NETs. Prospective studies about therapeutic strategies, integrating several treatments, should be performed to correlate therapeutic algorithms to specific clinical settings.

\section{Acknowledgements}

None.

\section{Footnote}

Conflicts of Interest: The authors have no conflicts of interest to declare.

\section{References}

1. Travis WD, Brambilla E, Müller-Hermelink HK, et al. editors. Tumours of the lung, pleura, thymus and heart. Lyon, France: IARC Press, 2004.

2. Travis WD, Giroux DJ, Chansky K, et al. International Staging Committee and Participating Institutions: The IASLC Lung Cancer Staging Project: proposals for the inclusion of broncho- pulmonary carcinoid tumors in the forthcoming (seventh) edition of the TNM Classification 
for Lung Cancer. J Thorac Oncol 2008;3:1213-23.

3. Öberg K, Hellman P, Ferolla P, et al. Neuroendocrine bronchial and thymic tumors: ESMO Clinical Practice Guidelines for diagnosis, treatment and follow-up. Ann Oncol 2012;23:vii120-123.

4. Rekhtman N. Neuroendocrine tumors of the lung: an update. Arch Pathol Lab Med 2010;134:1628-38.

5. Pelosi G, Fabbri A, Cossa M, et al. What clinicians are asking pathologists when dealing with lung neuroendocrineneoplasms? Seminars in diagnostic pathology 2015;32:469-79.

6. Rindi G, Arnold R, Bosman FT, et al. Nomenclature and classification of neuroendocrine neoplasms of the digestive system. In: Bosman FT, Carneiro F, Hruban RH, et al. editors. WHO Classification of Tumours of the Digestive System. Lyon: IARC Press, 2010:13-4.

7. Caplin ME, Baudin E, Ferolla P, et al. ENETS consensus conference participants. Pulmonary neuroendocrine (carcinoid) tumors: European Neuroendocrine Tumor Society expert consensus and recommendations for best practice for typical and atypical pulmonary carcinoids. Ann Oncol 2015;26:1604-20.

8. Wirth LJ, Carter MR, Jänne PA, et al. Outcome of patients with pulmonary carcinoid tumors receiving chemotherapy or chemoradiotherapy. Lung Cancer 2004;44:213-20.

9. Travis WD, Brambilla E, Burke AP, et al. editors. WHO Classification of Tumours of the Lung, Pleura, Thymus and Heart, 4th revised ed. Lyon: International Agency for Research on Cancer, World Health Organization, 2015.

10. Pelosi G, Pattini L, Morana G, et al. Lung neuroendocrine tumors: Controversies in search of a solution. Histol Histopathol 2017;32:223-41.

11. Haug AR, Cindea-Drimus R, Auernhammer CJ, et al. Neuroendocrine tumor recurrence: diagnosis with 68GaDOTATATE PET/CT. Radiology 2014;270:517-25.

12. Putzer D, Gabriel M, Henninger B, et al. Bone metastases in patients with neuroendocrine tumor: 68Ga-DOTATyr3-octreotide PET in comparison to CT and bone scintigraphy. J Nucl Med 2009;50:1214-21.

13. Lococo F, Perotti G, Cardillo G, et al. Multicenter comparison of 18F-FDG \& and 68Ga-DOTA-peptide PET/CT for pulmonary carcinoid. Clin Nucl Med 2015;40:e183-e189.

14. Gasparri R, Rezende GC, Fazio N, et al. Fluorodeoxyglucose positron emission tomography in pulmonary carcinoid tumors. Q J Nucl Med Mol Imaging 2015;59:446-54.

15. Pericleous M, Karpathakis A, Toumpanakis C, et al. Well- differentiated bronchial neuroendocrine tumors: Clinical management and outcomes in 105 patients. Clin Respir J 2016. [Epub ahead of print].

16. Ferolla P, Brizzi MP, Meyer T, et al. Efficacy and safety of pasireotide LAR or everolimus alone, or in combination in patients with advanced carcinoids (NET) of the lung/ thymus: Results from the randomized, phase 2 LUNA study. Ann Oncol 2016;27:vi136-vi148.

17. Yao JC, Fazio N, Singh S, et al. Everolimus for the treatment of advanced, non-functional neuroendocrine tumours of the lung or gastrointestinal tract (RADIANT-4): A randomised, placebo-controlled, phase 3 study. Lancet 2016;387:968-77.

18. Fazio N, Granberg D, Grossman A, et al. Everolimus plus octreotide long-acting repeatable in patients with advanced lung neuroendocrine tumors: analysis of the phase 3 , randomized, placebo-controlled RADIANT-2 study. Chest 2013;143:955-62.

19. Bajetta E, Catena L, Fazio N, et al. Everolimus in combination with octreotide long-acting repeatable in a first-line setting for patients with neuroendocrine tumors: an ITMO group study. Cancer 2014;120:2457-63.

20. Pavel ME, Wiedenmann B, Capdevila J, et al. RAMSETE: a single-arm, multicenter, single-stage phase II trial of RAD001 (everolimus) in advanced and metastatic silent neuro-endocrine tumours in Europe. J Clin Oncol 2012;30:abstr 4122.

21. Mariniello A, Bodei L, Tinelli C, et al. Long-term results of PRRT in advanced bronchopulmonary carcinoid. Eur J Nucl Med Mol Imaging 2016;43:441-52.

22. Imhof A, Brunner P, Marincek N, et al. Response, survival, and long-term toxicity after therapy with the radiolabeled somatostatin analogue [90YDOTA]-TOC in metastasized neuroendocrine cancers. J Clin Oncol 2011;29:2416-23.

23. Bodei L, Cremonesi M, Grana C, et al. Receptor radionuclide therapy with 90Y-[DOTA]0-Tyr3-octreotide (90Y-DOTATOC) in neuroendocrine tumours. Eur J Nucl Med Mol Imaging 2004;31:1038-46.

24. Ianniello A, Sansovini M, Severi S, et al. Peptide receptor radionuclide therapy with $177 \mathrm{Lu}-\mathrm{DOTATATE}$ in advanced bronchial carcinoids: prognostic role of thyroid transcription factor 1 and 18F-FDG PET. Eur J Nucl Med Mol Imaging 2016;43:1040-6.

25. Chong CR, Wirth LJ, Nishino M, et al. Chemotherapy for locally advanced and metastatic pulmonary carcinoid tumors. Lung Cancer 2014;86:241-6.

26. Walter T, Planchard D, Bouledrak K, et al. Evaluation of the combination of oxaliplatin and 5-fluorouracil 
or gemcitabine in patients with sporadic metastatic pulmonary carcinoid tumors. Lung Cancer 2016;96:68-73.

27. Bajetta E, Catena L, Procopio G, et al. Are capecitabine and oxaliplatin (XELOX) suitable treatments for progressing low-grade and high-grade neuroendocrine tumors? Cancer Chemother Pharmacol 2007;59:637-42.

28. Spada F, Antonuzzo L, Marconcini R, et al. OxaliplatinBased Chemotherapy in Advanced Neuroendocrine Tumors: Clinical Outcomes and Preliminary Correlation with Biological Factors. Neuroendocrinology 2016;103:806-14.

29. Crona J, Fanola I, Lindholm DP, et al. Effect of temozolomide in patients with meta- static bronchial carcinoids. Neuroendocrinology 2013;98:151-5.

30. Chan JA, Stuart K, Earle CC, et al. Prospective study of bevacizumab plus temozolomide in patients with advanced neuroendocrine tumors. J Clin Oncol 2012;30:2963-8.

31. Kunz PL, Balise RR, Fehrenbacher L, et al. OxaliplatinFluoropyrimidine Chemotherapy Plus Bevacizumab in Advanced Neuroendocrine Tumors: An Analysis of 2 Phase II Trials. Pancreas 2016;45:1394-400.

32. Saranga-Perry V, Morse B, Centeno B et al. Treatment of metastatic neuroendocrine tumors of the thymus with capecitabine and temozolomide: a case series. Neuroendocrinology 2013;97:318-21.

33. Crona J, Björklund P, Welin S, et al. Treatment, prognostic markers and survival in thymic neuroendocrine tumours. A study from a single tertiary referral centre. Lung Cancer 2013;79:289-93.
Cite this article as: Fazio N, Ungaro A, Spada F, Cella CA, Pisa E, Barberis M, Grana C, Zerini D, Bertani E, Ribero D, Funicelli L, Bonomo G, Ravizza D, Guarize J, De Marinis F, Petrella F, Del Signore E, Pelosi G, Spaggiari L. The role of multimodal treatment in patients with advanced lung neuroendocrine tumors. J Thorac Dis 2017;9(Suppl 15):S1501S1510. doi: 10.21037/jtd.2017.06.14 